
\title{
COVER
}

\section{Plutonium Release from Pressed Plutonium Oxide Fuel Pellets in Aquatic Environments}

\section{MASTER
ational Laboratory
ew Mexico 85545}




\section{DISCLAIMER}

This report was prepared as an account of work sponsored by an agency of the United States Government. Neither the United States Government nor any agency Thereof, nor any of their employees, makes any warranty, express or implied, or assumes any legal liability or responsibility for the accuracy, completeness, or usefulness of any information, apparatus, product, or process disclosed, or represents that its use would not infringe privately owned rights. Reference herein to any specific commercial product, process, or service by trade name, trademark, manufacturer, or otherwise does not necessarily constitute or imply its endorsement, recommendation, or favoring by the United States Government or any agency thereof. The views and opinions of authors expressed herein do not necessarily state or reflect those of the United States Government or any agency thereof. 


\section{DISCLAIMER}

Portions of this document may be illegible in electronic image products. Images are produced from the best available original document. 


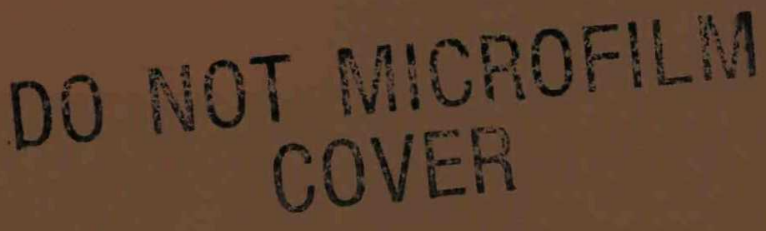

This work was supported by the US Department of Energy, Office of Special Nuclear Projects.

This report was prepared as an account of work sponsored by an agency of the United States Government. Neither the United States Government nor any agency thereof, nor any of their employees, makes any warranty, express or implied, or assumes any legal liability or responsibility for the accuracy, completeness. or usefulness of any information, apparatus, product, or process disclosed, or represents that its use would not infringe privately owned rights. Reference herein to any specific commercial product, process, or service by trade name, trademark, manufacturer, or otherwise, does not necessarily constitute or imply its endorsement, recommendation, or favoring by the United States Government or any agency thereof. The views and opinions of authors expressed herein do not necessarily state or reflect those of the United States Government or any agency thereof. 
UC-33a

HIIIF

Issued: December 1983

Pon?:gys

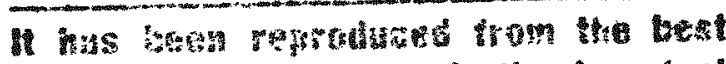

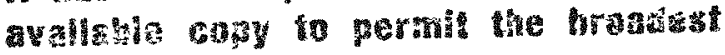

possible avallability.

$2 A--9962-115$

DE84 006692

\title{
Plutonium Release from Pressed Plutonium Oxide Fuel Pellets in Aquatic Environments
}

\author{
J. H. Patterson \\ F. J. Steinkruger \\ G. M. Matlack \\ R. C. Heaton \\ K. P. Coffelt \\ B. Herrera
}

\section{DISCLAIMER}

This report was prepared as an account of work sponsored by an agency of the United States Government. Neither the United States Government nor any agency thereof, nor any of their employees, makes any warranty, express or implied, or assumes any legal liability or responsibility for the accuracy, completeness, or usefulness of any information, apparatus, product, or process disclosed, or represents that its use would not infringe privately owned rights. Reference herein to any specific commercial product, process, or service by trade name, trademark, manufacturer, or otherwise does not necessarily constitute or imply its endorsement, recommendation, or favoring by the United States Government or any agency thereof. The views and opinions of authors expressed herein do not necessarily state or reflect those of the United States Government or any agency thereof.
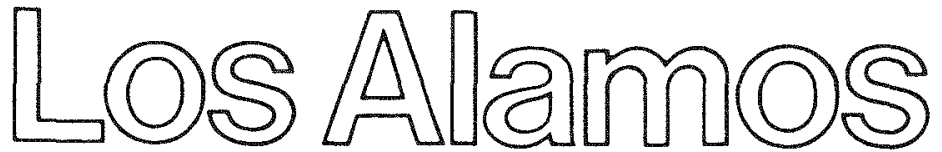


\title{
PLUTONIUM RELEASE FROM PRESSED PLUTONIUM OXIDE FUEL PELLETS IN AQUATIC ENVIRONMENTS
}

by

\author{
J. H. Patterson, F. J. Steinkruger, \\ G. M. Matlack, R. C. Heaton, \\ K. P. Coffelt, and B. Herrera
}

\begin{abstract}
Plutonium oxide pellets $\left(80 \%{ }^{238} \mathrm{Pu}, 40 \mathrm{~g}\right.$ each) were exposed to fresh water and sea water at two temperatures for $3 \mathrm{yr}$ in enclosed glass chambers. The concentrations of plutonium observed in the waters increased linearly with time throughout the experiment. However, the observed release rates were inversely dependent on temperature and salinity, ranging from $160 \mu \mathrm{Ci} /$ day for cold fresh water to $1.4 \mu \mathrm{Ci} /$ day for warm sea water. The total releases, including the chamber residues, showed similar dependencies. A major portion (typically greater than $50 \%$ ) of the released plutonium passed through a $0.1-\mu \mathrm{m}$ filter, with even larger fractions (greater than $80 \%$ ) for the fresh water systems.
\end{abstract}

\section{INTRODUCTION}

Radioisotope thermoelectric generators (RTGs) have been used extensively to provide auxiliary power in spacecraft. The radioisotope used almost exclusively in the US space program is ${ }^{238} \mathrm{Pu}$. In recent missions the plutonium has been in the form of plutonium dioxide $\left(\mathrm{PuO}_{2}\right)$ hot-pressed from powder. The heat source container, which was designed to remain intact under the heat of orbital reentry and impact with earth, has been extensively tested. However, the Department of Energy (DOE) continually seeks more information about potential plutonium release modes and the possible effects of released plutonium in order to improve the safety of the heat sources and to provide data for use in risk assessment studies. As part of this program, the Los Alamos National Laboratory is studying the interactions of ${ }^{238} \mathrm{PuO}_{2}$ fuel materials with various environmental systems.

Interactions of bare and clad fuel material with terrestrial environments have been reported, ${ }^{1-4}$ as have previous experiments with plutonium dioxide in aqueous systems. ${ }^{9-7}$ Earlier aquatic experiments indicated that the apparent dissolution rate of plutonium dioxide varied inversely with temperature and salinity. ${ }^{8}$ The present experiments were carried out to obtain absolute release rates in aqueous media in order to understand the cause of this paradoxical behavior. Most of the previous aquatic experiments used aquaria whose plastic-coated plywood sides and bottoms were not conducive to recovery of precipitated or sorbed plutonium. In the present experiment an all-glass apparatus was used, so that quantitative recovery could be achieved.

\section{I. EXPERIMENTAL SECTION}

The glass chambers used were cylindrical, measuring 12 in. long and 6 in. in diameter (Fig. 1). They were constructed with two pieces of 6 -in. flanged pyrex pipe with the flanges situated in the middle of the cylinder. The two sections were held together with a 6-in. 


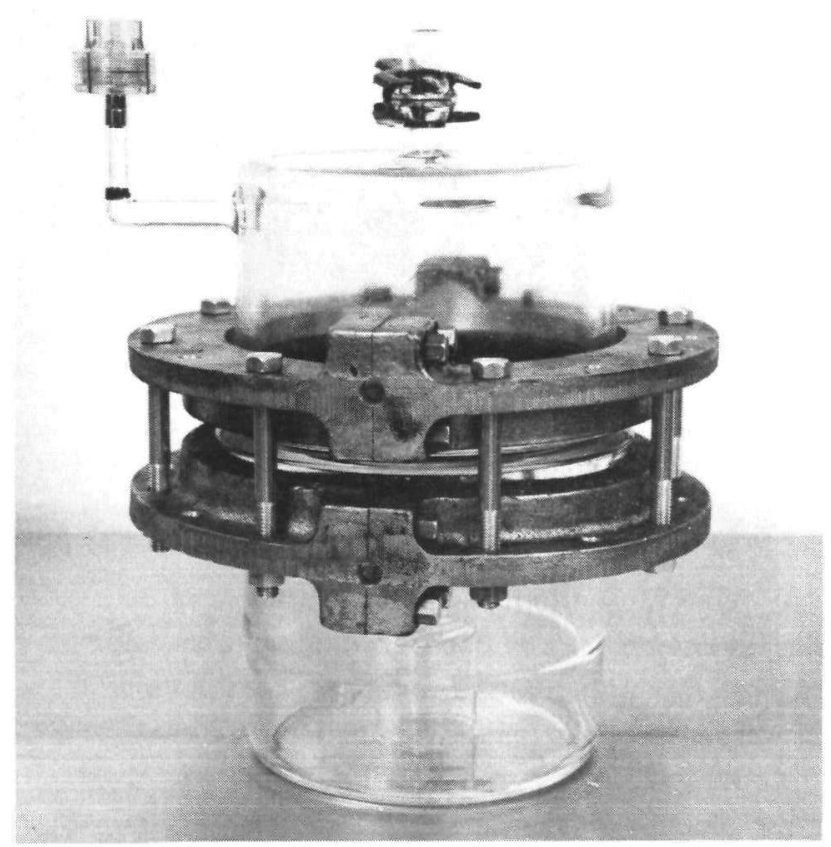

Fig. 1. A glass chamber.

aluminum flange set and a neoprene gasket. The ends of the cylinder were sealed and flattened to resemble the bottom of a beaker. The top of each cylinder included a 28/15 o-ring joint through which was inserted a fritted glass gas-dispersion tube. In addition, a side arm was located near the top on the side of each cylinder. This was constructed with 10 -mm o.d. glass and was approximately 2 in. long. A plastic filter holder containing a filter $(0.3 \mu \mathrm{m}$ or less) was affixed to this side arm so that air, admitted to the chamber through the gas-dispersion tube, could exit without carrying radioactive particles into the room air. A small pedestal, 2 in. high and 2 in. in diameter, was placed in the bottom of each cylinder. The plutonium oxide fuel pellet was placed on this pedestal at the beginning of the experiment.

Temperature control was accomplished by maintaining the glass chambers in thermostatted water baths (Aquarium Systems, Inc., model CS 30). Each water bath had a capacity of 30 gallons. Two such baths were used, one maintained at $10^{\circ} \mathrm{C}$ and the other at $35^{\circ} \mathrm{C}$. Each bath contained two chambers, one with fresh water (distilled water) and one with simulated sea water

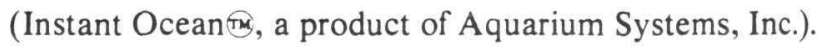

The sources used were pressed plutonium oxide (PPO) fuel pellets, $40 \mathrm{~g}$ each, manufactured at the Los Alamos National Laboratory. The plutonium used was $80 \%$ ${ }^{238} \mathrm{Pu}$. The pellet identification numbers were HPZ-201-2, HPZ-201-3, HPZ-203-1, and HPZ-203-4.

At the beginning of the experiment, the bottom of each chamber was filled with $2 \mathrm{~L}$ of the appropriate aqueous medium, and the fuel pellet was placed on the pedestal. The top was clamped in place, and the chamber was secured in the temperature bath. Agitation within each chamber was accomplished by bubbling air through the liquid. Because the water level within the chambers was maintained below the flange seal, the liquid and headspace volumes were approximately the same. The water levels within the chambers were kept constant by periodic additions of water to the chambers. Water samples were withdrawn weekly by removing the gas-dispersion tube and inserting a 1.0 - or $0.5-\mathrm{mL}$ volumetric pipet through the joint. At the conclusion of the experiment, the chambers were opened and the sources removed. The aqueous contents were sequentially filtered through filters of decreasing pore size, and the filters and filtrates were analyzed for plutonium. The $\mathrm{pH}$ of the aqueous media was measured before filtration. In addition, the residues were leached from the chambers with dilute acid, and the residues along with the leachates were analyzed for plutonium.

The residues and filters were dissolved by digestion with nitric and hydrofluoric acids. Aliquots of these solutions, as well as water from the chambers, were analyzed for plutonium by mixing them with scintillation cocktail and counting the scintillations with a Packard Tricarb सxis.

\section{RESULTS AND DISCUSSION}

The concentrations of plutonium observed in the water phases of the chambers are shown in Fig. 2 and in Table A-I of the Appendix. These values were determined by measuring all the plutonium in aliquots taken from the chambers and include soluble as well as suspended material. Because the water volumes were approximately constant at $2 \mathrm{~L}$, the absolute releases can be obtained by multiplying the concentrations by 2 . The results clearly show that the concentration of plutonium increases linearly with time, which suggests that the release rate from each source is constant. The slopes of the curves are shown in Table I. Note that the largest slope, and thus the greatest release rate $(160 \mu \mathrm{Ci} / \mathrm{day})$, was observed for cold fresh water, and that the lowest slope, 


\section{SEALED CHAMBER DATA}

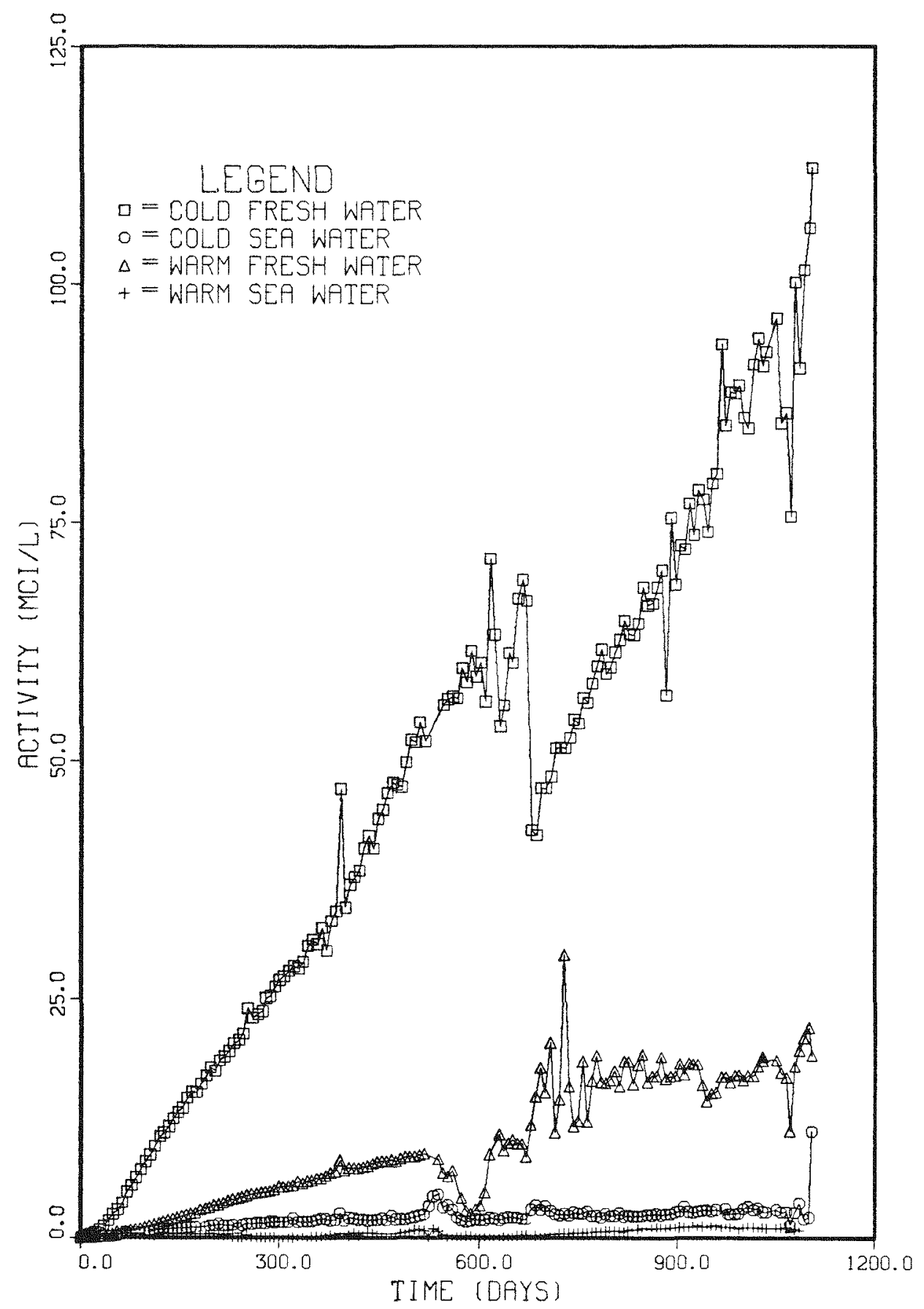

Fig. 2. The observed plutonium releases versus time; the water volumes were nominally $2.0 \mathrm{~L}$. 


\begin{tabular}{|c|c|c|c|}
\hline Cold & Cold & Warm & Warm \\
\hline Fresh & Sea & Fresh & Sea \\
\hline Water & Water & Water & Water \\
\hline 160 & 2.9 & 28 & 1.4 \\
\hline
\end{tabular}

and thus the lowest release rate $(1.4 \mu \mathrm{Ci} /$ day $)$, was observed for warm sea water. In general, the observed release rates decrease with increasing temperature and are lower for sea water than for fresh water at a given temperature, consistent with the results from related aquarium experiments. ${ }^{8}$

The primary reason for implementing this experiment with enclosed glass chambers was to permit determination of the total release, including that material deposited on the sides and bottom of the chamber. Accordingly, after removing the sources, the aqueous contents of the chambers were decanted and characterized, and the residues were washed out of the chambers with dilute acid. The results of these characterizations are listed in Table A-II and summarized in Tables II through IV.

Table II shows the total plutonium releases obtained from the final chamber inventories. These data suggest that the amount of plutonium in the residue, when compared with the total, is significantly larger for the sea water than for the fresh water. However, the total release shows the same trends as does the apparent aqueous release. Consequently, the differences in the observed aqueous releases cannot be explained by invoking hypothetical subsequent chemical reactions to remove released plutonium by incorporating it into the sediment. The inverse temperature dependence of the release rate indicates that plutonium release from the source is not a straightforward, thermally controlled process. Furthermore, the fact that the observed release rates are different for the two media suggests that the source is not the sole determining factor for the release rate. There does not appear to be a straightforward relationship between the release rate and $\mathrm{pH}$. Although the highest release occurs at the lowest $\mathrm{pH}$, as one might expect, the lowest release rate occurs at the next lowest $\mathrm{pH}$ value.

Note that the final aqueous total for the cold sea water (Table II) is substantially larger than one would predict from the previously observed aqueous release rates (Table I). This increase also can be seen in the last data point in Fig. 2. Because both sets of data seem to be internally consistent, we ignored the final data point in calculating the aqueous release rate (Table I) but used it (and ignored the previous data points) in evaluating the final chamber inventories (Tables II through IV and AII). We have no reasonable explanation for this anomalous behavior.

After removing the water from the chambers, aliquots were taken and filtered sequentially through $0.45-\mu \mathrm{m}$ Millipore and $0.1-\mu \mathrm{m}$ Unipore filters. We designated this as Series 1 (Table III). After 1 month, the remaining samples were filtered through a $0.4-\mu \mathrm{m}$ Nuclepore filter. An aliquot of each filtrate then was filtered through a 0.1- $\mu \mathrm{m}$ Uniporea filter. We designated this as Series 2 (Table IV). The Uniporem and Nucleporem filters are both polycarbonate membrane filters, which have very narrow pore size distributions. The Milliporem filters are mat-type filters made from cellulose esters. These have a much wider range of pore sizes than do the polycarbonate membranes, so the particle sizes that pass through this type of filter are much less well defined.

Tables III and IV show that significant amounts (usually more than $50 \%$ ) of the observed activity passes through the finest filter used $(0.1 \mu \mathrm{m})$. This is especially true in the case of fresh water, in which more than $80 \%$ passed through the finest filter. For the coarse filters, larger percentages of the activity were retained from sea water than from fresh water. Note that the nature of the particles retained is not known. These larger particles may be plutonium oxide or they may consist of other materials carrying plutonium. The larger retentions for the sea water are consistent with the larger amounts of plutonium in the residue observed for these samples. There appears to be little temperature dependence on the percentages retained by the various filters, except possibly for the material passing the coarse filters and remaining on the fine filter. These observations suggest that a major portion of the released plutonium exists in the form of particles (or other forms) less than $0.1 \mu \mathrm{m}$ in size. It is possible that nearly all particles are less than this size and that some are carried by larger particles of other materials. Determining the mechanism of plutonium release would require more thorough characterization of the released activity and of other potential controlling factors.

\section{SUMMARY}

Pellets of ${ }^{238} \mathrm{PuO}_{2}$ (40 g each) were exposed to fresh water and sea water at two temperatures for $3 \mathrm{yr}$. The concentration of plutonium observed in the waters 


\begin{tabular}{lllll}
\hline \hline \multicolumn{1}{l}{ TABLE II. Final Chamber Inventories } & & \\
\hline & Cold & Cold & Warm & Warm \\
& Fresh & Sea & Fresh & Sea \\
& Water & Water & Water & Water \\
\hline Aqueous total $(\mathrm{mCi})$ & 220 & 30 & 47 & 2.7 \\
Residue total $(\mathrm{mCi})$ & 45.7 & 47.0 & 11.9 & 2.7 \\
Total release $(\mathrm{mCi})$ & 270 & 77 & 59 & 5.4 \\
Final pH & 3.85 & 8.3 & 6.67 & 5.75 \\
\hline \hline
\end{tabular}

increased linearly with time, which suggests that the rates of release of plutonium from the pellets were constant over the duration of the experiment. However, the observed rates were inversely dependent on temperature and salinity, ranging from $160 \mu \mathrm{Ci} /$ day for cold fresh water to $1.4 \mu \mathrm{Ci} /$ day for warm sea water. The total releases, including the chamber residues, showed similar dependencies, indicating that these differences cannot be explained by removal of plutonium from the water phase by precipitations or other chemical reactions. There was no clear correlation of the release with $\mathrm{pH}$ of the final solution.

TABLE III. Results of Aqueous Filtrations-Series 1

\begin{tabular}{|c|c|c|c|c|}
\hline & $\begin{array}{l}\text { Cold } \\
\text { Fresh } \\
\text { Water }\end{array}$ & $\begin{array}{l}\text { Cold } \\
\text { Sea } \\
\text { Water }\end{array}$ & $\begin{array}{l}\text { Warm } \\
\text { Fresh } \\
\text { Water }\end{array}$ & $\begin{array}{l}\text { Warm } \\
\text { Sea } \\
\text { Water }\end{array}$ \\
\hline$\%$ retained on $0.45-\mu \mathrm{m}$ filter ${ }^{a}$ & 0.11 & $83-100$ & 3.6 & 12 \\
\hline$\%$ retained on $0.1-\mu \mathrm{m}$ filter ${ }^{b}$ & 0.05 & 0.07 & 0.03 & 0.08 \\
\hline$\%$ passing $0.1-\mu \mathrm{m}$ filter ${ }^{b}$ & $>99$ & $0-17$ & 96 & 88 \\
\hline
\end{tabular}

"Millipore filter; filtrations were carried out immediately after completion of the experiment. bUniporem filter; the sample used was the filtrate from the $0.45-\mu \mathrm{m}$ filter.

TABLE IV. Results of Aqueous Filtrations-Series 2

\begin{tabular}{lcccc}
\hline & $\begin{array}{l}\text { Cold } \\
\text { Fresh } \\
\text { Water }\end{array}$ & $\begin{array}{l}\text { Cold } \\
\text { Sea } \\
\text { Water }\end{array}$ & $\begin{array}{l}\text { Warm } \\
\text { Fresh } \\
\text { Water }\end{array}$ & $\begin{array}{l}\text { Warm } \\
\text { Sea } \\
\text { Water }\end{array}$ \\
\hline${\text { \% retained on } 0.4-\mu \mathrm{m} \text { filter }^{\mathrm{a}}}^{\text {\% retained on } 0.1-\mu \mathrm{m}_{\text {filter }}{ }^{\mathrm{b}}}$ & 5.9 & 45 & 6.7 & 49 \\
\% passing $0.1-\mu \mathrm{m}$ filter $^{\mathrm{b}}$ & 83 & 6.4 & 0.02 & 0.02 \\
Final $\mathrm{pH}$ & 3.85 & 49 & 93 & 51 \\
\hline
\end{tabular}

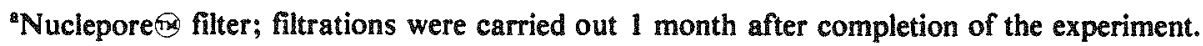

bUnipores filter; the sample used was the filtrate from the $0.4-\mu \mathrm{m}$ filter.

${ }^{c}$ Calculated by difference because the sample was lost. 
A major portion (typically greater than $50 \%$ ) of the released plutonium passed through a $0.1-\mu \mathrm{m}$ filter, with even larger fractions (greater than $80 \%$ ) for the fresh water systems. Thus, virtually all the plutonium released may be in a very fine form, with some being carried by other solid material. Elucidation of the release mechanism will require further experiments involving more thorough characterization of the aqueous media and the released plutonium.

\section{ACKNOWLEDGMENTS}

We wish to thank Evangeline Hodge, Gilbert B. Nelson, Joseph Bubernak, and Margaret Romero for performing the plutonium analyses necessary for this work. We are also grateful to Henry Kavanaugh and Delbert Romero for performing the filtrations at the conclusion of the experiment.

\section{REFERENCES}

1. J. H. Patterson, B. Herrera, G. B. Nelson, G. M. Matlack, and G. R. Waterbury, "Interaction of an Iridium-Clad RTG Heat Source Unit with a Simulated Terrestrial Environment," Los Alamos Scientific Laboratory report LA-6255 (April 1976).

2. F. J. Steinkruger, J. H. Patterson, B. Herrera, G. B. Nelson, G. M. Matlack, G. R. Waterbury, and D. Pavone, "Interaction of a ${ }^{238} \mathrm{Pu}$ Fueled Sphere Assembly with a Simulated Terrestrial Environment," Los Alamos National Laboratory report LA-8597 (February 1981).
3. J. H. Patterson, B. Herrera, G. B. Nelson, F. J. Steinkruger, G. M. Matlack, and D. Pavone, "LongTerm Exposure of ${ }^{238} \mathrm{PuO}_{2}$ to a Terrestrial Environment," Los Alamos National Laboratory report LA-9487-MS (December 1982).

4. J. H. Patterson, R. C. Heaton, B. Herrera, G. B. Nelson, and F. J. Steinkruger, "Long-Term Exposure of ${ }^{238} \mathrm{PuO}_{2}$ to a Terrestrial Environment," Los Alamos National Laboratory report LA-9487-MS, Vol. II (September 1983).

5. J. H. Patterson, G. B. Nelson, and G. M. Matlack, "The Dissolution of ${ }^{238} \mathrm{Pu}$ in Environmental and Biological Systems," Los Alamos Scientific Laboratory report LA-5624 (July 1974).

6. G. M. Matlack, J. H. Patterson, G. B. Nelson, and G. R. Waterbury, "Dissolution Rates of ${ }^{238} \mathrm{PuO}_{2}$ and ${ }^{239} \mathrm{PuO}_{2}$ in $1 M$ Perchloric Acid," Los Alamos Scientific Laboratory report LA-6184 (March 1976).

7 G. M. Matlack, G. B. Nelson, J. H. Patterson, and G. R. Waterbury, "Plutonium Release from PMC Heat Sources in Aquatic Environments," Los Alamos Scientific Laboratory report LA-6423 (October 1976).

8. G. R. Waterbury, Compiler, "Environmental and Radiological Safety Studies, April 1-June 30, 1976. Interaction of ${ }^{238} \mathrm{PuO}_{2}$ Heat Sources with Terrestrial and Aquatic Environments," Los Alamos Scientific Laboratory report LA-6507-PR (September 1976). 
APPENDIX. EXPERIMENTAL DATA

TABLE A-I. Observed Aqueous Plutonium Concentrations

\begin{tabular}{|c|c|c|c|c|c|c|c|c|c|}
\hline \multirow[b]{2}{*}{ ample } & \multirow[b]{2}{*}{$\begin{array}{l}\text { date } \\
\text { rmod }\end{array}$} & \multicolumn{2}{|c|}{$\begin{array}{l}\text { chamber } 18 a \\
\text { cold fresh vater }\end{array}$} & \multicolumn{2}{|c|}{$\begin{array}{l}\text { Chamber } 18 \mathrm{~b} \\
\text { cold sea bater }\end{array}$} & \multicolumn{2}{|c|}{$\begin{array}{l}\text { chamber } 19 a \\
\text { warm fresh water }\end{array}$} & \multicolumn{2}{|c|}{$\begin{array}{ll}\text { Chamber } & 19 \mathrm{~b} \\
\text { warm sea } & \text { ater }\end{array}$} \\
\hline & & $\begin{array}{c}\text { activity } \\
(c i / 1) \\
-0 . .-\end{array}$ & $\begin{array}{l}\text { error } \\
\text { (cill }\end{array}$ & $\begin{array}{l}\text { activitu } \\
(c, i t) \\
\cdots-\ldots . .\end{array}$ & $\begin{array}{l}\text { error } \\
\text { (cilil) }\end{array}$ & $\begin{array}{c}\text { activity } \\
(c i, 1) \\
-\ldots \ldots\end{array}$ & $\begin{array}{l}\text { error } \\
\text { rci, } 1) \\
\ldots \ldots \ldots\end{array}$ & $\begin{array}{c}\text { activity } \\
(c i, j) \\
\ldots \ldots . . .\end{array}$ & $\begin{array}{l}\text { error } \\
\text { (ci } 1) \\
\ldots . .2 .\end{array}$ \\
\hline 1 & 800221 & $4760 e-04$ & $2260 e-c 4$ & $\cdots$ & - & - & $\cdots$ & $\cdots$ & 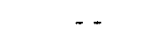 \\
\hline 2 & 800222 & 3900 e- 04 & 4100 e- 05 & $2750 e-04$ & 4000 e- 06 & 1980 e-04 & 9000 e-05 & $2470 e-05$ & $0000-06$ \\
\hline 3 & 800225 & $8000 e-04$ & 1500 e-05 & $2980 e-04$ & & & & $3990 e-04$ & \\
\hline 4 & 800227 & $.1200 e-03$ & $1000 e-06$ & $.32500-04$ & $30000-06$ & $.5480 e-04$ & $31402-04$ & $4860 e-04$ & $3000 e-c 6$ \\
\hline 5 & 800229 & $1.000-03$ & $5000 e-06$ & $.37300-04$ & 5000 e-06 & .4920 e -04 & $3300 e-05$ & $.64909-04$ & 9000 e-06 \\
\hline 6 & 03 & .03 & 05 & 04 & 05 & 8780 & -04 & $.96 \triangle O S$ & $2200 e-05$ \\
\hline$i$ & & 29006 & 210 & 688 & & 4 & 5 & 1363 & -04 \\
\hline 8 & 80 & $3800 e-03$ & $5800 e-05$ & .8590 & $1140 e-04$ & .8270 e-04 & $1180 e-04$ & $.308 e-03$ & -05 \\
\hline 9 & 800 & $4500-03$ & 4000 - 05 & $.1034 e-03$ & $22200-04$ & $.1183 e-03$ & $4300 e-05$ & $.1650 e-03$ & $40000-06$ \\
\hline 10 & $80 r$ & $6200 e-03$ & -04 & .1100 & & $1543 e-03$ & -05 & $2126 e-03$ & .06 \\
\hline$t$ & & 03 & 04 & 130 & 28 & 20096 & 5 & 269 & .06 \\
\hline & & & 80 & +90 & & 5202 & & 3 & \\
\hline 1 & 80 & 1960 & 290 & .2000 & .77 & .3464 & 730 & 3971 & -04 \\
\hline 14 & 80 & $2500 e-02$ & 4000 & .2000 & .770 & .42726 & -05 & .4190 & -05 \\
\hline 15 & & .02 & 04 & .2800 & 461 & $.7172 €$ & 332 & $.3941 e-0.3$ & -05 \\
\hline 16 & 3 & 2 & 4 & 4 & 18 & 3 & b & 3 & \\
\hline 47 & 30 & 477 & 170 & .6000 & 57 & 6876 & .05 & .4002 & 05 \\
\hline 19 & 80 & $5450 e$ & 20 & .4000 & 38 & 7951 & -04 & .3557 & .05 \\
\hline 19 & & 02 & & .500 & 18 & .8826 & 04 & .3432 & -05 \\
\hline 20 & 800 & $.7110 e-02$ & $.87000-04$ & .5600 & .17 & $.9828 €$ & $e-04$ & $.2942 e-03$ & -05 \\
\hline 2 & & $.7960 \mathrm{e}$ & 04 & 57 & 15 & 02 & 05 & & -05 \\
\hline & & .869 & 50 & .630 & & 1230 & o & & 05 \\
\hline 23 & 80 & .960 & & .750 & & 1380 & .05 & .2857 & -05 \\
\hline 24 & 80 & .105 & 14 & .860 & & .1530 & -05 & .4336 & -05 \\
\hline 25 & 80 & $.1099 e-01$ & .5400 e- 04 & $.9100 e-03$ & .43 & .1700 e-02 & $1000 e-05$ & $.4881 e-03$ & -05 \\
\hline 26 & & 01 & 04 & & & 02 & 05 & 03 & -06 \\
\hline & 80 & 12 & 43 & .93 & & 2 & 05 & .374 & 05 \\
\hline & & 131 & 38 & .98 & & .21 & 05 & .3625 & .06 \\
\hline 29 & & .135 & 94 & & & & .05 & & 06 \\
\hline 30 & 80 & $.4464 \mathrm{e}-01$ & 6100 & .104 & 30 & .2400 & .04 & .3648 & 05 \\
\hline 3 & & & 93 & 10 & & & & & \\
\hline & & & 33 & 2 & & & & .34 & \\
\hline & & .16 & .64 & .1 & & 02 & 04 & .35 & .05 \\
\hline 34 & & & & $.92-32-x$ & & & 04 & .38 & -04 \\
\hline & & .17816 & .80 & .1260 & .24 & .31 & .04 & .4396 & -05 \\
\hline & & & & & & & & & \\
\hline 37 & & & & & & & & & -06 \\
\hline 20 & & .189 & & .12 & & .36 & .7 & & $=-05$ \\
\hline & & .19 & 40 & .133 & & .388 & .380 & .358 & \\
\hline & 80 & 202 & 968 & 9900 & & $.4010 \mathrm{e}-02$ & $e-04$ & .1087 & 3000 e-0 \\
\hline 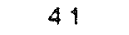 & & .20 & & & & & & 58 & -06 \\
\hline & & & & & & & & & \\
\hline & & .240 & & .14 & & .442 & .450 & 28 & $D E$ \\
\hline & 80 & .2302 & & .152 & & .45 & 04 & 352 & -05 \\
\hline & & $.2341 e-01$ & 03 & 1530 & & .4650 & -0 & 04 & $1003 e-05$ \\
\hline & & & & & & & & & \\
\hline & & .25 & 03 & 169 & & .480 & 820 & 38 & 5 \\
\hline & 801 & $.2532 e-01$ & De-03 & $.1580 e-02$ & & 4890 & $2100 e-04$ & $355 \mathrm{Ce}-04$ & $e .06$ \\
\hline 49 & 801210 & $.2629 e-01$ & $1700 e-03$ & .1680 & & 50506 & $5500 e-04$ & 4510 & $9-C 5$ \\
\hline (5) & 801217 & $9 e-01$ & $2900 e-03$ & .1640 e-02 & $.9800 e-04$ & $53009-02$ & $0 e-04$ & $21900-04$ & $2630 e-04$ \\
\hline
\end{tabular}


TABLE A-I. (cont)

\begin{tabular}{|c|c|c|c|c|c|c|c|c|c|}
\hline $\begin{array}{l}51 \\
52 \\
52 \\
54\end{array}$ & $\begin{array}{l}801223 \\
801231 \\
810107 \\
810114 \\
810124\end{array}$ & $\begin{array}{l}2739 e-01 \\
2795 e-01 \\
2241 \mathrm{e}-01 \\
2820 \mathrm{e}-01 \\
2888 \mathrm{e}-01\end{array}$ & $\begin{array}{l}4500 e-04 \\
2500 e-04 \\
4800 e-04 \\
8600 e-03 \\
9000 e-04\end{array}$ & $\begin{array}{l}1650 \mathrm{e}-02 \\
1640 \mathrm{e}-02 \\
2020 \mathrm{e}-02 \\
1540 \mathrm{e}-02 \\
1680 \mathrm{e}-\mathrm{c} 2\end{array}$ & $\begin{array}{l}4700 e-04 \\
2300 e-04 \\
2370 e-03 \\
3600 e-04 \\
4600 e-04\end{array}$ & $\begin{array}{l}5340 e-02 \\
5410 e-02 \\
5500-02 \\
58300-02 \\
5680 e-02\end{array}$ & $\begin{array}{l}7200 e-04 \\
35700-04 \\
68000-04 \\
20300-03 \\
23000-04\end{array}$ & $\begin{array}{l}41100-04 \\
40500-04 \\
41000-04 \\
4520 e-04 \\
-9300-04\end{array}$ & $\begin{array}{l}16000 \text { o5 } \\
22020-35 \\
29000-05 \\
26000-05 \\
6200 e \text { o }\end{array}$ \\
\hline $\begin{array}{l}56 \\
57 \\
58 \\
59 \\
60\end{array}$ & $\begin{array}{l}810128 \\
810204 \\
810211 \\
810218 \\
810225\end{array}$ & $\begin{array}{l}3057 e-01 \\
3121 e-01 \\
3075 e-01 \\
3243 e-01 \\
3007 e-01\end{array}$ & $\begin{array}{l}6000 e-05 \\
2680 e-02 \\
9100 e-03 \\
3890=03 \\
2430 e-03\end{array}$ & $\begin{array}{l}1680 \text { - } 02 \\
1700 \text { e } 02 \\
186 \text { e }-02 \\
1090-02 \\
1950 \text { e- } 02\end{array}$ & $\begin{array}{r}3000 e-04 \\
1400 e-04 \\
1130 e-03 \\
6000 e-05 \\
.2600 e-04\end{array}$ & $\begin{array}{l}5940 e-02 \\
6000 e-02 \\
6190 e-02 \\
6220 e-02 \\
6480 e-02\end{array}$ & $\begin{array}{l}1100 e-04 \\
1700 e-04 \\
1630 e-03 \\
4100 e-04 \\
1000 e-05\end{array}$ & $\begin{array}{l}9630 e-04 \\
1216 e-03 \\
1272 e-03 \\
1638 e-03 \\
2098 e-03\end{array}$ & $\begin{array}{l}1400 e-05 \\
1000 e-05 \\
-000 e-05 \\
2-00 e-05 \\
2300 e-05\end{array}$ \\
\hline $\begin{array}{l}61 \\
62 \\
53 \\
64 \\
65\end{array}$ & $\begin{array}{l}810304 \\
810311 \\
810318 \\
810325 \\
810401\end{array}$ & $\begin{array}{l}3317 e-01 \\
3416 e-01 \\
4700 e-01 \\
3456 e-01 \\
3696 e-01\end{array}$ & $\begin{array}{l}16.90 e-03 \\
1000 e-03 \\
2052 e-02 \\
6000 e-03 \\
4090 e-03\end{array}$ & $\begin{array}{l}1780 \mathrm{e}-02 \\
1890 \mathrm{e}-02 \\
2590 \mathrm{e}-02 \\
1820 \mathrm{e}-02 \\
2110 \mathrm{e}-02\end{array}$ & $\begin{array}{r}1000 \text { e-04 } \\
1050 e-03 \\
1750 \text { e }-03 \\
1100 \text { e }-04 \\
1670 \text { e }-03\end{array}$ & $\begin{array}{l}6770 \mathrm{e}-02 \\
5930 \mathrm{e}-02 \\
8110 \mathrm{e}-02 \\
7080 \mathrm{e}-02 \\
.7310 \mathrm{e}-02\end{array}$ & $\begin{array}{c}7200 e-04 \\
1100 e-04 \\
2110 e-03 \\
1490 e-03 \\
--\end{array}$ & $\begin{array}{l}2579 e-03 \\
3149 e-03 \\
4474 e-03 \\
4175 e-03 \\
2270 e-03\end{array}$ & $\begin{array}{l}6000 \\
7000 \\
1460\end{array}$ \\
\hline $\begin{array}{l}66 \\
67 \\
68 \\
69 \\
70\end{array}$ & $\begin{array}{l}810408 \\
810415 \\
810422 \\
810429 \\
810505\end{array}$ & $\begin{array}{l}3777 e-01 \\
3844 e-01 \\
4080 e-01 \\
4208 e-01 \\
4075 e-01\end{array}$ & $\begin{array}{r}5000 e-05 \\
1123 e-02 \\
1360 e-02 \\
5390 e-03 \\
6000 e-04\end{array}$ & $\begin{array}{l}2 c 10 e-02 \\
1880 e-02 \\
1930 e-02 \\
1810 e-02 \\
1870 e-02\end{array}$ & $\begin{array}{r}1510 e-03 \\
.9500 e-04 \\
4500 e-04 \\
3700 e-04 \\
.6000 e-04\end{array}$ & $\begin{array}{l}7310 e-02 \\
7310 e-02 \\
7420 e-02 \\
7530 e-02 \\
7800 e-02\end{array}$ & $\begin{array}{l}2660 e-03 \\
1500 e-04 \\
1080 e-03 \\
62000-04 \\
1490 e-03\end{array}$ & $\begin{array}{l}4867 e-03 \\
2569 e-03 \\
5110 e-03 \\
5299 e-03 \\
4885 e-03\end{array}$ & 1000 \\
\hline $\begin{array}{l}71 \\
72 \\
73 \\
74 \\
75\end{array}$ & $\begin{array}{l}810513 \\
810520 \\
810527 \\
810504 \\
810610\end{array}$ & $\begin{array}{l}4390 e-01 \\
4484 e-01 \\
4661 e-01 \\
4766 e-01 \\
4745 e-01\end{array}$ & $\begin{array}{r}4720 e-03 \\
5990 e-03 \\
3020 e-03 \\
8450 e-03 \\
1570 e-03\end{array}$ & $\begin{array}{l}1880 e-02 \\
2030 e-02 \\
1860 e-02 \\
2320 e-02 \\
1950 e-02\end{array}$ & $\begin{array}{r}4400 e-04 \\
7900 e-04 \\
4700 e-04 \\
1340 e-03 \\
1260 e-03\end{array}$ & $\begin{array}{l}7970 \mathrm{e}-02 \\
8050 \mathrm{e}-02 \\
8040 \mathrm{e}-02 \\
8190 \mathrm{e}-02 \\
802 \mathrm{Ce}-02\end{array}$ & $\begin{array}{l}3100 e-04 \\
5000 e-05 \\
1370 e-03 \\
29800-03 \\
3700 e-04\end{array}$ & $\begin{array}{l}4579 e-02 \\
14530-03 \\
4235 e-03 \\
43-5 a-03 \\
43240-03\end{array}$ & $\begin{array}{l}-05 \\
-05 \\
-05 \\
-04\end{array}$ \\
\hline $\begin{array}{l}76 \\
77 \\
78 \\
79\end{array}$ & $\begin{array}{l}810 \\
810 \\
810 \\
810 \\
810\end{array}$ & $\begin{array}{l}e-01 \\
e-01 \\
e-01 \\
e-01 \\
e-01\end{array}$ & $\begin{array}{l}e-02 \\
e-03 \\
e-03 \\
e-06 \\
e-05\end{array}$ & $\begin{array}{l}e-c 2 \\
\text { e- } 02 \\
\text { e- } 02 \\
e-02 \\
e-02\end{array}$ & $\begin{array}{l}\text { ee }-03 \\
\text { be } 04 \\
\text { be-04 } \\
\text { be-04 } \\
\text { be-04 }\end{array}$ & $\begin{array}{l}e-02 \\
e-02 \\
e-02 \\
e-02 \\
e-c 2\end{array}$ & $\begin{array}{l}0 \text { ee-04 } \\
0 e-04 \\
0 e-03 \\
0 e-05 \\
0 e-04\end{array}$ & $\begin{array}{l}5159 e-c 3 \\
5134 e-03 \\
6059 e-03 \\
826 c e-03 \\
849-0-03\end{array}$ & $\begin{array}{l}2-200-04 \\
4800 e-05 \\
1000 e-04 \\
72000-05\end{array}$ \\
\hline $\begin{array}{l}81 \\
82 \\
83 \\
84 \\
85\end{array}$ & $\begin{array}{l}810722 \\
810729 \\
810805 \\
810812 \\
810819\end{array}$ & $\begin{array}{c}.5200 \mathrm{e}-01 \\
\ldots \\
\cdots \\
.5580-01\end{array}$ & $\begin{array}{c}.1000 \text { e-06 } \\
-- \\
- \\
- \\
.8000-06\end{array}$ & $\begin{array}{l}.2430 e-02 \\
.3300 e-02 \\
.4350 e-02 \\
.4530 e-02 \\
.3190 e-02\end{array}$ & $\begin{array}{l}.1680 e-03 \\
.8880 e-03 \\
.7200 e-04 \\
.7900 e-04 \\
.7400 e-04\end{array}$ & $\begin{array}{c}.8780 \mathrm{e}-02 \\
\ldots \\
.8200 \mathrm{e}-02 \\
.6790 \mathrm{e}-02\end{array}$ & $\begin{array}{c}.8300 \mathrm{e}-04 \\
-. \\
.5200-04 \\
.7650 \mathrm{e}-03\end{array}$ & $\begin{array}{l}7538 e-03 \\
1131 e-03 \\
9149 e-03 \\
6900 e-03 \\
3286 e-03\end{array}$ & $\begin{array}{r}1050 e-04 \\
.1410 e-04 \\
2300 e \text { e5 } \\
.7700 \text { e-05 } \\
.3100 \text { e- } 05\end{array}$ \\
\hline $\begin{array}{l}86 \\
87 \\
88 \\
89 \\
90\end{array}$ & $\begin{array}{l}810902 \\
810909 \\
810916 \\
810923\end{array}$ & $\begin{array}{l}.5640 \mathrm{e}-01 \\
.5670 \mathrm{e}-01 \\
.5650 \mathrm{e}-01 \\
.5970 \mathrm{e}-01 \\
.5820 \mathrm{e}-01\end{array}$ & $\begin{array}{l}.1500 e-05 \\
.2000 \text { e-06 } \\
.1200 \text { e-05 } \\
.8000 \text { e-06 } \\
.5000 \text { - } 06\end{array}$ & $\begin{array}{l}.3410 \mathrm{e}-02 \\
.2940 \mathrm{e}-02 \\
.2130 \mathrm{e}-02 \\
.1860 \mathrm{e}-02 \\
.1690 \mathrm{e}-02\end{array}$ & $\begin{array}{l}.1970 e-03 \\
.1590 e-03 \\
.8200 e-04 \\
.6900 e-04 \\
.4000 e-04\end{array}$ & $\begin{array}{c}6390 e-02 \\
7040 e-02 \\
-- \\
4140 e-02 \\
2820 e-02\end{array}$ & $\begin{array}{c}1000 \mathrm{e}-05 \\
.1310 \mathrm{e}-03 \\
-- \\
.1060 \mathrm{e}-03 \\
.1000 \mathrm{e}-05\end{array}$ & $\begin{array}{l}1418 e-03 \\
2055 e-03 \\
2039 e-03 \\
2117 e-03 \\
2195 e-03\end{array}$ & $\begin{array}{l}\text { be-04 } \\
\text { be-04 } \\
\text { be-05 } \\
\text { be-05 } \\
\text { be-04 }\end{array}$ \\
\hline $\begin{array}{l}91 \\
92 \\
93 \\
94 \\
95\end{array}$ & $\begin{array}{l}810930 \\
811007 \\
811014 \\
811021 \\
811028\end{array}$ & $\begin{array}{l}.6150 e-01 \\
.5880 e-01 \\
.6030 e-01 \\
.5620 e-01 \\
.7120 e-01\end{array}$ & $\begin{array}{l}.9000 e-06 \\
.7000 e-06 \\
.1900 \text { e-05 } \\
.3100 \text { e-05 } \\
.4800 \text { e-05 }\end{array}$ & $\begin{array}{l}1790 e-02 \\
.1970 \text { e-02 } \\
.1830 e-02 \\
.1890 e-02 \\
.2090 \text { e }-02\end{array}$ & $\begin{array}{l}.1900 e-04 \\
.2000 e-04 \\
.1510 e-03 \\
.2500 e-04 \\
.1140 e-03\end{array}$ & $\begin{array}{l}.2570 e-02 \\
.2990 e-02 \\
.3380 e-02 \\
.4750 e-02 \\
.8750 e-02\end{array}$ & $\begin{array}{r}.1100 e-04 \\
.7000 \text { e-05 } \\
5300 \text { - } 04 \\
.1800 \text { e- } 04 \\
.8200 \text { e- } 04\end{array}$ & $\begin{array}{l}2031 \mathrm{e}-03 \\
1921 \mathrm{e}-03 \\
1897 \mathrm{e}-03 \\
1947 \mathrm{e}-03 \\
2000 \mathrm{e}-03\end{array}$ & $\begin{array}{l}.3000 e-05 \\
.1200 e-05 \\
.6000 e-06 \\
.2700 e-05 \\
.3100 e-05\end{array}$ \\
\hline $\begin{array}{l}96 \\
97 \\
98 \\
99\end{array}$ & 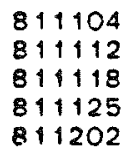 & $\begin{array}{l}.6320 \mathrm{e}-01 \\
.5360 \mathrm{e}-01 \\
.5580 \mathrm{e}-01 \\
.6130 \mathrm{e}-01 \\
.6030 \mathrm{e}-01\end{array}$ & $\begin{array}{l}.1220 e-05 \\
.3300 e-05 \\
.4100 e-05 \\
.2400 e-05 \\
.2000 \text { e- } 05\end{array}$ & $\begin{array}{l}.2030 \mathrm{e}-02 \\
.1870 \mathrm{e}-02 \\
.2050 \mathrm{e}-02 \\
.2170 \mathrm{e}-02 \\
.2150 \mathrm{e}-02\end{array}$ & $\begin{array}{l}.6300 e-04 \\
.1200 e-04 \\
.8000 e-05 \\
.5600 e-04 \\
.8100 e-04\end{array}$ & $\begin{array}{c}1082 \text { e-01 } \\
.9150 \text { e-02 } \\
.9940 e-02 \\
.1030 e-01\end{array}$ & $\begin{array}{c}-- \\
.3560 \text { e-03 } \\
.5800 \mathrm{e}-04 \\
.7600 \mathrm{e}-04 \\
.3670 \mathrm{e}-03\end{array}$ & $\begin{array}{l}2069 \\
1668 \\
2064 \\
2220 \\
1780\end{array}$ & $\begin{array}{l}.05 \\
.05 \\
05\end{array}$ \\
\hline 0 & $\begin{array}{l}811209 \\
811216 \\
811222 \\
811230 \\
820106\end{array}$ & $\begin{array}{l}.6700 \mathrm{e}-01 \\
.6900 \mathrm{e}-01 \\
.6680 \mathrm{e}-01 \\
.4270 \mathrm{e}-01 \\
.4220 \mathrm{e}-01\end{array}$ & $\begin{array}{l}.1200 e-05 \\
.1000 \text { e- } 05 \\
.3000 \text { e- } 06 \\
.3200 \text { e }-05 \\
.4000 \text { e- } 06\end{array}$ & $\begin{array}{l}.214 \\
.198 \\
.198 \\
.296 \\
.343\end{array}$ & $\begin{array}{l}.6000 e-05 \\
.8000 e-05 \\
.2600 e-04 \\
.8050 e-03 \\
.1650 e-04\end{array}$ & $\begin{array}{l}9880 e-02 \\
.9880 e-02 \\
.8490 e-02 \\
1182-01 \\
1480 e-01\end{array}$ & $\begin{array}{l}0 e-04 \\
0 e-04 \\
0 e-04 \\
2 e-02 \\
0 e-04\end{array}$ & $\begin{array}{l}2396 e-03 \\
2477 e-03 \\
2622 e-03 \\
2242 e-03 \\
2238 e-03\end{array}$ & $\begin{array}{l}.90 \\
.21 \\
.86 \\
.61\end{array}$ \\
\hline 11 & $\begin{array}{l}820120 \\
820128 \\
820204 \\
820211\end{array}$ & $\begin{array}{l}4710 \text { e- } 01 \\
.4830 \text { e }-01 \\
.5130 \text { e }-01 \\
.5140 \text { e }-01\end{array}$ & $\begin{array}{l}1600 \text { e-05 } \\
6000 \text { e-06 } \\
6000 \text { e }-06 \\
1000 \text { e }-06 \\
6000 \text { e }-06\end{array}$ & $\begin{array}{l}3380 \text { e }-02 \\
.2880 \text { e }-02 \\
.2500 \text { e }-02 \\
.2360 \text { e }-02\end{array}$ & $\begin{array}{l}.3900-04 \\
.3300 e-04 \\
.1520 e-03 \\
.1140 e-03 \\
.3900 e-04\end{array}$ & $\begin{array}{l}1781 e-01 \\
1527 e-01 \\
.2046 e-01 \\
1107 e-01 \\
1454 e-01\end{array}$ & $\begin{array}{l}.4720 \text { e-03 } \\
.7900 \text { - } 04 \\
.6500 \text { e-04 } \\
.2920 \text { e-03 }\end{array}$ & $\begin{array}{l}3135 e-03 \\
3265 e-03 \\
3816 e-03 \\
3812 e-03 \\
4126 e-03\end{array}$ & $\begin{array}{l}21 \\
.36 \\
.39\end{array}$ \\
\hline
\end{tabular}


TABLE A-I. (cont)

\begin{tabular}{|c|c|c|c|c|c|c|c|c|c|}
\hline $\begin{array}{l}111 \\
112 \\
113 \\
114 \\
115\end{array}$ & $\begin{array}{l}820218 \\
820225 \\
820304 \\
820311 \\
820318\end{array}$ & $\begin{array}{l}5130 e-01 \\
5240 e-01 \\
5430 e-01 \\
5380 e-01 \\
5660 e-01\end{array}$ & $\begin{array}{r}4000 e-06 \\
1300 e-05 \\
7000 e-06 \\
1500 e-05 \\
.3000 e-06\end{array}$ & $\begin{array}{r}2560 \mathrm{e}-02 \\
2340 \mathrm{e}-02 \\
.2670 \mathrm{e}-02 \\
.2560 \mathrm{e}-02 \\
.2570 \mathrm{e}-02\end{array}$ & $\begin{array}{r}.2610 e-03 \\
.1200 e-04 \\
.1290 e-03 \\
3200 e-04 \\
4300 e-04\end{array}$ & $\begin{array}{l}2966 e-01 \\
1587 e-01 \\
1172 e-01 \\
1230 e-01 \\
1851 e-01\end{array}$ & $\begin{array}{r}4000 e-03 \\
2900 e-04 \\
2960 e-03 \\
4870 e-03 \\
8000 e-04\end{array}$ & $\begin{array}{r}4378 e-03 \\
4715 e-03 \\
4792 e-03 \\
5205 e-03 \\
5273 e-03\end{array}$ & $\begin{array}{l}4500 e-05 \\
5290 e-05 \\
3900 e-05 \\
1290 e-04 \\
1000 e-04\end{array}$ \\
\hline $\begin{array}{l}116 \\
117 \\
118 \\
119 \\
120\end{array}$ & $\begin{array}{l}820325 \\
820401 \\
820408 \\
820415 \\
820422\end{array}$ & $\begin{array}{r}5610 e-01 \\
5810 e-01 \\
5990 e-01 \\
6170 e-01 \\
5910 e-01\end{array}$ & $\begin{array}{r}6000 \text { e-06 } \\
.3000 \text { e-06 } \\
6000 \text { e-06 } \\
3300 \text { e- } 05 \\
.1000 \text { e }-05\end{array}$ & $\begin{array}{r}.2810 e-02 \\
2270 e-02 \\
2320 e-02 \\
.2160 e-02 \\
.2550 e-02\end{array}$ & $\begin{array}{r}4700 e-04 \\
.8400 e-04 \\
1200 e-04 \\
.4900 e-04 \\
.1160 e-03\end{array}$ & $\begin{array}{l}1247 \mathrm{e}-01 \\
1650 \mathrm{e}-01 \\
1913 \mathrm{e}-01 \\
1635 \mathrm{e}-01 \\
1623 \mathrm{e}-01\end{array}$ & $\begin{array}{l}2580 \mathrm{e}-03 \\
1790 \mathrm{e}-03 \\
1690 \mathrm{e}-03 \\
1600 \mathrm{e}-04 \\
2800 \mathrm{e}-04\end{array}$ & $\begin{array}{r}5676 e-03 \\
5894 e-03 \\
6290 e-03 \\
6235 e-03 \\
.6437 e-03\end{array}$ & $\begin{array}{l}8500 e-05 \\
1650 e-04 \\
1800 e-05 \\
1400 e-05 \\
1010 e-04\end{array}$ \\
\hline $\begin{array}{l}121 \\
122 \\
123 \\
124 \\
125\end{array}$ & $\begin{array}{l}820429 \\
820506 \\
820513 \\
820520 \\
820527\end{array}$ & $\begin{array}{r}.5980 e-01 \\
.61400-01 \\
.6270 e-01 \\
6470 e-01 \\
.6330 e-01\end{array}$ & $\begin{array}{r}1800 e-05 \\
3180 \text { e- } 04 \\
7000 \text { e }-06 \\
5000 \text { e }-06 \\
8000 \text { e }-06\end{array}$ & $\begin{array}{r}.2300 e-02 \\
2330-02 \\
.2600 e-02 \\
.2240 e-02 \\
.2350 e-02\end{array}$ & $\begin{array}{l}.1180 \text { e-03 } \\
.2170 \text { e-03 } \\
.9000 \text { e-05 } \\
.1100 \text { e-04 } \\
.1100 \text { e }-03\end{array}$ & $\begin{array}{l}.1657 e-01 \\
.1751 e-01 \\
.1589 e-01 \\
.1851 e-01 \\
.1849 e-01\end{array}$ & $\begin{array}{l}2650 e-03 \\
7200 e-04 \\
4340 e-03 \\
2930 e-03 \\
.8243 e-02\end{array}$ & $\begin{array}{r}7387 e-03 \\
7296 e-03 \\
.7231 e-03 \\
6109 e-03 \\
.7702 e-03\end{array}$ & $\begin{array}{r}1350 e-04 \\
3650 e-04 \\
8500 e-05 \\
1430 e-04 \\
1440 e-04\end{array}$ \\
\hline $\begin{array}{l}126 \\
127 \\
128 \\
129 \\
130\end{array}$ & $\begin{array}{l}820503 \\
820610 \\
820617 \\
820624 \\
820701\end{array}$ & $\begin{array}{l}6320 \mathrm{e}-01 \\
6440 \mathrm{e}-01 \\
6820 \mathrm{e}-01 \\
6630 \mathrm{e}-01 \\
6650 \mathrm{e}-01\end{array}$ & $\begin{array}{l}1000 e-06 \\
3600 e-05 \\
1500 e-05 \\
4500 e-05 \\
9000 e-05\end{array}$ & $\begin{array}{l}2280 e-02 \\
2300 e-02 \\
2280 e-02 \\
2440 e-02 \\
2380 e-02\end{array}$ & $\begin{array}{l}5300 e-04 \\
1290 e-03 \\
7000 e-05 \\
3300 e-04 \\
2300 e-04\end{array}$ & $\begin{array}{l}1611 \mathrm{e}-01 \\
1815 e-01 \\
1922 e-01 \\
1632 e-01 \\
1698 e-01\end{array}$ & $\begin{array}{l}15-0 e-03 \\
3900 e-04 \\
1382 e-02 \\
1960 e-03 \\
5700 e-04\end{array}$ & $\begin{array}{l}8542 e 03 \\
8954 \text { e } 03 \\
1000 \text { e } 02 \\
1020 e-02 \\
9500 e-03\end{array}$ & $\begin{array}{l}E \text { E } 70 e-05 \\
5100 e-05 \\
5500 e-04 \\
1000 e-04 \\
5000 e-05\end{array}$ \\
\hline $\begin{array}{l}131 \\
132 \\
133 \\
134 \\
135\end{array}$ & $\begin{array}{l}820708 \\
820715 \\
820722 \\
820729 \\
820805\end{array}$ & $\begin{array}{r}6820 e-01 \\
7000 e-01 \\
.5690 e-01 \\
7550 e-01 \\
6850 e-01\end{array}$ & $\begin{array}{l}1400 \text { e }-05 \\
8000 \text { e } 06 \\
3600 \text { e } 05 \\
1000 \text { e }-06 \\
5700 \text { e }-05\end{array}$ & $\begin{array}{l}2530 \mathrm{e}-\mathrm{O} 2 \\
2380 \mathrm{e}-02 \\
2490 \mathrm{e}-02 \\
2500 \mathrm{e}-02 \\
2690 \mathrm{e}-02\end{array}$ & $\begin{array}{l}8100 e-04 \\
3400 e-04 \\
9600 e-04 \\
9500 e-04 \\
5300 e-04\end{array}$ & $\begin{array}{l}1699 e-01 \\
1899 e-01 \\
1669 e-01 \\
1696 e-01 \\
1695 e-01\end{array}$ & $\begin{array}{l}4220 e-03 \\
8650 e-03 \\
5630 e-03 \\
8000 e-04 \\
3950 e-03\end{array}$ & $\begin{array}{l}1010 \text { e } 02 \\
9900 \text { e-03 } \\
1050 \text { e-02 } \\
1110 \text { e } 02 \\
1140 \text { e-02 }\end{array}$ & $\begin{array}{l}9000 e-05 \\
1400 e-04 \\
2600 e-04 \\
2100 e-04 \\
4800 e-04\end{array}$ \\
\hline $\begin{array}{l}136 \\
137 \\
138 \\
139 \\
140\end{array}$ & $\begin{array}{l}820812 \\
820819 \\
820826 \\
820902 \\
820909\end{array}$ & $\begin{array}{l}-01 \\
-01 \\
-01 \\
-01 \\
-01\end{array}$ & $\begin{array}{l}2400 \text { e-05 } \\
9000 \text { e-06 } \\
1400 \text { e }-05 \\
1400 \text { - } 05 \\
5000 \text { e }-06\end{array}$ & $\begin{array}{l}2780 e-02 \\
3280 \text { e }-02 \\
2740 e-02 \\
2630 e-02 \\
2770 \text { e } 02\end{array}$ & $\begin{array}{l}-03 \\
-03 \\
-03 \\
-03 \\
-04\end{array}$ & $\begin{array}{l}1828 e-01 \\
1712 e-01 \\
1834 e-01 \\
1823 e-01 \\
1818 e-01\end{array}$ & $\begin{array}{l}9000 e-04 \\
6500 e-04 \\
1810 e-03 \\
4530 \text { e } 03 \\
3820 \text { e }-03\end{array}$ & $\begin{array}{l}1100 e-02 \\
11400-02 \\
1160 e-02 \\
11300-02 \\
1270 e-02\end{array}$ & $\begin{array}{l}0 e-05 \\
0 e-04 \\
0 e-05 \\
0 e-05 \\
0 e-04\end{array}$ \\
\hline $\begin{array}{l}141 \\
142 \\
143 \\
144 \\
145\end{array}$ & $\begin{array}{l}820916 \\
820923 \\
820930 \\
82100 \\
821015\end{array}$ & $\begin{array}{l}7740 e-01 \\
7400 e-01 \\
7910 e-01 \\
8010 e-01 \\
9370 e-01\end{array}$ & $\begin{array}{c}3000 \text { e } 06 \\
2400 \text { e } 05 \\
3000 \text { e } 06 \\
1850 \text { - } 04 \\
\ldots\end{array}$ & $\begin{array}{l}2870 e-02 \\
2920 e-02 \\
2810 e-02 \\
2960 \text { e } 02 \\
2520 e-02\end{array}$ & $\begin{array}{l}1390 e-03 \\
4500 e-04 \\
6200 e-04 \\
5100 e-04 \\
1830 e-03\end{array}$ & $\begin{array}{l}1604 e-01 \\
1433 e-01 \\
1516 e-01 \\
1527 e-01 \\
1688 e-01\end{array}$ & $\begin{array}{l}4990 \text { e }-03 \\
12-0 \text { e }-03 \\
1430 \text { e }-03 \\
6500 \text { - } 04 \\
1810 \text { e }-03\end{array}$ & $\begin{array}{l}1180 e-02 \\
1179 e-02 \\
1220 e-02 \\
12-0 e-02 \\
1170 e-02\end{array}$ & $\begin{array}{l}2100 e-04 \\
1200 e-04 \\
9300 e-04 \\
2000 e-05 \\
3000 e-05\end{array}$ \\
\hline $\begin{array}{l}146 \\
147 \\
148 \\
149 \\
150\end{array}$ & $\begin{array}{l}821021 \\
821028 \\
821104 \\
821110 \\
821117\end{array}$ & $\begin{array}{l}8520 e-01 \\
8870 e-01 \\
8860 e-01 \\
8940 e-01 \\
8600 e-01\end{array}$ & $\begin{array}{l}1600 e-05 \\
1400 e-05 \\
8000 e-06 \\
2500 e-05 \\
4000 e-05\end{array}$ & $\begin{array}{l}3050 e-02 \\
2500 e-02 \\
2500 e-02 \\
2500 e-02 \\
3070 e-02\end{array}$ & $\begin{array}{l}6000 e-04 \\
4100 e-04 \\
2800 e-04 \\
1010 e-03 \\
5600 e-04\end{array}$ & $\begin{array}{l}=-01 \\
=-01 \\
=-01 \\
=-01 \\
=-01\end{array}$ & $\begin{array}{l}7600 e-04 \\
6200 e-04 \\
2940 e-03 \\
2210 e-03 \\
1220 \text { e }-03\end{array}$ & $\begin{array}{l}1150 e-02 \\
1140 e-02 \\
1080 e-02 \\
1020 e-02 \\
1060 e-02\end{array}$ & $\begin{array}{l}2000 e-05 \\
1000 e-04 \\
2900 e-04 \\
1100 e-04 \\
3000 e-04\end{array}$ \\
\hline $\begin{array}{l}151 \\
152 \\
153 \\
154 \\
155\end{array}$ & $\begin{array}{l}821124 \\
821202 \\
821209 \\
821216 \\
821221\end{array}$ & $\begin{array}{l}8490 e-01 \\
9160 e-01 \\
9430 e-01 \\
9140 e-01 \\
9290 e-01\end{array}$ & $\begin{array}{l}4200 e-05 \\
1300 e-05 \\
1170 e-04 \\
1200 e-05 \\
4000 e-06\end{array}$ & $\begin{array}{l}3290 e-02 \\
2950 e-02 \\
3120 e-02 \\
2760 e-02 \\
2700 e-c 2\end{array}$ & $\begin{array}{l}2000 e-04 \\
8000 e-05 \\
1070 e-03 \\
1050 e-03 \\
1300 e-04\end{array}$ & $\begin{array}{l}1700 e-01 \\
1700 e-01 \\
1796 e-01 \\
1897 e-01 \\
1859 e-01\end{array}$ & $\begin{array}{l}2470 e-03 \\
7350=-03 \\
1302 e-02 \\
1590 e-03 \\
2580 e-n 3\end{array}$ & $\begin{array}{l}1100 e-02 \\
1080 e-02 \\
1100 e-02 \\
1030 e-02 \\
1030 e-02\end{array}$ & $\begin{array}{l}1000 e-04 \\
3000 e-05 \\
1000 e-05 \\
4000 e-04 \\
2000 e-05\end{array}$ \\
\hline $\begin{array}{l}156 \\
157 \\
158 \\
159 \\
160\end{array}$ & $\begin{array}{l}830105 \\
830113 \\
830121 \\
83012^{-} \\
830203\end{array}$ & $\begin{array}{l}9640 e-01 \\
8540 e-01 \\
8650 e-01 \\
7560 e-01 \\
1002 e+00\end{array}$ & $\begin{array}{l}1000 e-06 \\
6000 e-05 \\
3800 e-05 \\
8000 \text { e } 06 \\
1900 \text { e } 05\end{array}$ & $\begin{array}{l}2970 e-02 \\
2710 e-02 \\
2730 e-02 \\
1360 e-02 \\
2660 e-02\end{array}$ & $\begin{array}{l}7600 e-04 \\
2640 e-03 \\
2340 e+00 \\
2110 e-03 \\
9600 e-04\end{array}$ & $\begin{array}{l}1864 e-01 \\
1731 e-01 \\
1683 e-01 \\
1122 e-01 \\
1798 e-01\end{array}$ & $\begin{array}{l}6000 e-04 \\
3270 e-03 \\
6460 e-03 \\
1424 e-02 \\
6520 e-03\end{array}$ & $\begin{array}{l}9800 e-03 \\
1040 e-02 \\
1100 e-02 \\
6400 e-03 \\
9300 e-03\end{array}$ & $\begin{array}{l}1400 e-04 \\
5100 e-04 \\
3900=-04 \\
1000 e-05 \\
4900 e-04\end{array}$ \\
\hline $\begin{array}{l}161 \\
162 \\
163 \\
164\end{array}$ & $\begin{array}{l}830210 \\
830217 \\
830225 \\
830301\end{array}$ & $\begin{array}{l}9120 e-01 \\
1015 e+00 \\
1059 e+00 \\
1122 e+00\end{array}$ & $\begin{array}{l}1290 \text { e-04 } \\
3000 \text { e }-06 \\
1700 \text { - } 05 \\
6200 e-05\end{array}$ & $\begin{array}{l}\text { be }-02 \\
\text { be-02 } \\
\text { De-02 } \\
\text { be-04 }\end{array}$ & $\begin{array}{l}2170 e-03 \\
1870 e-03 \\
1290 e-03 \\
2706 e-02\end{array}$ & $\begin{array}{l}1962 e-01 \\
2098 e-01 \\
2205 e-01 \\
1911 e-01\end{array}$ & $\begin{array}{l}1950 \text { e-03 } \\
7930 \text { e-03 } \\
4200 e-04 \\
1641 e-02\end{array}$ & $\begin{array}{c}8300 e-03 \\
\ldots \\
\cdots\end{array}$ & $\begin{array}{c}23000-04 \\
--\end{array}$ \\
\hline
\end{tabular}

The source was introduced into chamber 18a on 800220

Sources vere introduced into the other chambers on 800221 
TABLE A-1I. Final Analyses of Sealed Chamber Contents

$18 \mathrm{~A}$

$18 \mathrm{~B}$

$19 \mathrm{~A}$

$19 B$

Cold Fresh Water Cold Sea Water Warm Fresh Water Warm Sea Water

1. Final aqueous total $(\mathrm{mCi})^{\mathrm{a}}$

2. Final aqueous volume (L)

3. Final residue total $(\mathrm{mCi})$

4. Total release $(\mathrm{mCi})$

5. Final $\mathrm{pH}$

6. $\mathrm{mCi}$ retained on $0.4-\mu \mathrm{m}$ filter ${ }^{\mathrm{b}}$

7. $\mathrm{mCi}$ in filtrate from $0.4-\mu \mathrm{m}$ filter $^{\mathrm{b}}$

8. Total volume filtered (L)

9. $\mu \mathrm{Ci}$ retained on $0.1-\mu \mathrm{m}$ filter ${ }^{\mathrm{c}}$

10. $\mathrm{mCi}$ in filtrate from $0.1-\mu \mathrm{m}$ filter

11. Volume filtered $(\mathrm{mL})$

12. $\mu \mathrm{Ci}$ retained on $0.45-\mu \mathrm{m}$ filter $\mathrm{e}^{\mathrm{e}}$

13. $\mu \mathrm{Ci}$ retained on $0.1-\mu \mathrm{m}$ filter $\mathrm{f}$

14. $\mathrm{mCi} / \mathrm{L}$ in filtrate from $0.1-\mu \mathrm{m}^{\text {filter }}{ }^{\mathrm{g}}$

$\begin{array}{cl}220 & \pm 20 \\ 2.0 & \\ 45.7 & \pm 0.6 \\ 270 & \pm 20 \\ 3.85 & \end{array}$

$11.1 \pm 0.1$
$176 \pm 0.8$
1.75
$560^{d}$

$4.47 \pm 0.02$

50.0

$6.0 \pm 0.2$

$2.98 \pm 0.04$

$103 \pm 1$

$\begin{array}{cl}30 & \pm 8 \\ 2.7 & \\ 47.0 & \pm 0.6 \\ 77 & \pm 8 \\ 8.3 & \end{array}$

$16.4 \pm 0.6$

$20.3 \pm 0.4$

2.0

$58.0 \pm 0.9$

$0.47 \pm 0.02$

50.0

$56.0 \pm 20$

$3.9 \pm 0.2$

$1.2 \pm 1$

$\begin{array}{clll}47 & \pm 6 & 2.7 & \pm 0.3 \\ 2.4 & & 2.3 & \\ 11.9 & \pm 0.4 & 2.7 & \pm 0.2 \\ 59 & \pm 6 & 5.4 & \pm 0.4 \\ 6.67 & & 5.75 & \\ 4.1 & \pm 0.2 & 1.32 & \pm 0.008 \\ 57.0 & \pm 0.3 & 2.43 & \pm 0.009 \\ 2.3 & & 1.70 & \\ 0.296 & \pm 0.002 & 0.0160 & \pm 0.0001 \\ 1.38 & \pm 0.08 & 0.0983 & \pm 0.0001 \\ 50.0 & & 50.0 & \end{array}$

$34.2 \pm 0.7$

$0.270 \pm 0.005$

$6.9 \pm 0.3$

$0.048 \pm 0.005$

$0.75 \pm 0.06$

'The specific activity of the sources was $13.649 \mathrm{Ci} / \mathrm{g}$.

'Nuclepore@ filter; the sample volume is listed in line 8.

'Unipore@ filter; the sample was taken from the filtrate from the 0.4- $\mu \mathrm{m}$ filter (line 7).

${ }^{\mathrm{d} C}$ alculated by difference because the sample was lost.

'Millipore@ filter; the sample used was a $50-\mathrm{mL}$ aliquot from the original aqueous sample (line 1).

Uniporem filter; the sample used was the filtrate from the previous line (line 12).

The filtrate volume was not measured. 


\section{DO NOT MICROFILM
COVER in. :}

Printed in the United States of America Available from

National Technical Information Service

US Department of Commerce

5285 Port Royal Road

Springfield. VA 2216

Microfiche (A0I)

NTIS

\begin{tabular}{cc} 
Page Range & Price Code \\
\hline 001.025 & $\mathrm{~A} 02$ \\
026.050 & $\mathrm{~A} 03$ \\
051.075 & $\mathrm{~A} 04$ \\
$076 \cdot 100$ & $\mathrm{~A} 05$ \\
101.125 & $\mathrm{~A} 06$ \\
126.150 & $\mathrm{~A} 07$
\end{tabular}

NTIS

Page Range

Price Code

151-175

176.200

201.225

226.250

251.275

276. 300
A08

$\mathrm{A} 09$

A 10

A 11

A12

Al3
NTIS

Page Range Price Code

301.325 Al4

326. 350

351.375

376.400

$401-425$

426.450
Al4

A 15

A 16

A 17

A 18
NTIS

Page Range Price Cod $451.475 \quad$ A20

A 21

$501.525 \quad$ A22

$526550 \quad$ A23

$551575 \quad$ A24

$576-600 \quad$ A25

601-up* A99

*Contact NTIS for a price quote. 


\section{DO NOT MICHOFLM COVER

Los Allamos 